P Castellanos Pineda, J Sprünker Cardó, G Munilla Cabrillana (2016): "Inmigrantes y museos de ciencias en tiempo de crisis. Estudio preliminar en dos museos de Barcelona". Revista Latina de Comunicación Social, 71, pp. 570 a 591

http://www.revistalatinacs.org/071/paper/1110/30es.html

DOI: $\underline{10.4185 / R L C S-2016-1110}$

\title{
Inmigrantes y museos de ciencias en tiempo de crisis. Estudio preliminar en dos museos de Barcelona
}

\section{Immigrants and science museums in times of crisis. Preliminary study in two museums in Barcelona}

Patricia Castellanos Pineda [CV] [DORCID] [ GGS] Estudios de Comunicación. Estudios Art y Humanidades. Universitat Oberta de Catalunya (UOC) pcastellanos@uoc.edu

Janine Sprünker Cardó [CV] [ $[$ ORCID] [CGS] Centre d'Idiomes Moderns. Estudios Art y Humanidades. Universitat Oberta de Catalunya (UOC) jsprunker@uoc.edu

Gloria Munilla Cabrillana [ $[\underline{\mathrm{CV}}]$ [ORCID] [ $\mathrm{GGS}$ ] Estudios Art y Humanidades. Universitat Oberta de Catalunya (UOC) gmunilla@uoc.edu

\begin{abstract}
s
[ES] Introducción: El acceso al conocimiento genera desigualdades. El abismo que se produce entre los grupos alfabetizados científicamente y los que no, es susceptible de convertirse en causa de exclusión. En España, la relación entre los museos de ciencia y la población vulnerable, en este caso, la población inmigrante, ha sido poco trabajada, por lo que se busca contribuir a una línea consolidada en otros países. Metodología: El estudio utilizó encuestas en dos museos de ciencias de Barcelona para: conocer el perfil sociodemográfico, la relación que establece el público inmigrante con el museo y, sondear, la relación museo e identidad en dos museos de Barcelona. Resultados: Se comprobó que los visitantes se sienten cómodos en el museo, y encuentran elementos expositivos comunes entre su país y los del museo. En la interpretación de los resultados debe tenerse en cuenta que se trata de un estudio previo.

[EN] Introduction: Access to knowledge generates inequalities. The gap between those who are scientifically literate and those who are not can become a source of exclusion. In Spain, the relationship between science museums and vulnerable communities, in this case the immigrant population has been little studied, and we are therefore seeking to build on research developed in other countries. Methodology: The study used surveys to gain an understanding of interviewees'
\end{abstract}


socio-demographic profile, how immigrants relate to museums and the relationship between the museum and identity in two museums in Barcelona. Results: Visitors consulted stated that they felt comfortable in the museum and found the display formats in the museum to be similar to those in their own countries. It must be borne in mind that this is a preliminary study.

\section{Keywords}

[ES] Inmigrantes; museos de ciencias como medios de comunicación; inclusión social; museos y público vulnerable

[EN] Migrants, science museums as mass media, social inclusion, museums and vulnerable public

\section{Contents}

[ES] 1. Introducción. 1.1. La exclusión y la inclusión. 1.2. Museos e inclusión. 1.3. Museo e identidad. 2. Método. 2.1. Participantes. 2.2. Estrategias metodológicas. 2.3. Procedimientos. 3. Resultados. 3.1. Cosmocaixa. 3.2. Museo Marítimo 4. Discusión y conclusiones. 5. Notas. 6. Bibliografía.

[EN] 1. Introduction. 1.1. Exclusion and Inclusion. 1.2. Museum and inclusion. 1.3. Museum and identity. 2. Method. 2.1. Participants. 2.2. Methodological Strategies. 2.3. Processes. 3. Findings. 3.1. Cosmocaixa. 3.2. Museum Marítimo 4. Discussion and conclusions. 5.Notes. 6. Bibliography.

Traducción realizada por el Servicio Lingüístico de la Universitat Oberta de Catalunya (UOC)

\section{Introducción}

A continuación se exponen los resultados de dos estudios preliminares [1] en torno al proyecto de investigación: "Ciencia e inclusión social en el entorno de museos y centros de ciencias. Los inmigrantes y los MCyT en Catalunya" realizada en el Museo Marítimo de Barcelona y Cosmocaixa de Barcelona, con la colaboración del grupo de investigación Museia (Elearn Centre, UOC).

La investigación tiene como objetivo principal conocer la relación que tienen los inmigrantes con los museos de ciencias. Como objetivos específicos se planteó averiguar el perfil sociodemográfico del visitante e indagar en los aspectos del museo que el usuario identifica como propios o diferentes a su entorno de nacimiento en la visita al museo, gracias a las preguntas abiertas de la encuesta.

Partimos de la concepción del museo como medio de comunicación teniendo en cuenta que a finales de la década de los 60 cuando el canadiense Duncan Cameron publica el artículo A view point: the museum as a communication system and implications for museum education, en el que desarrolla el tema. Cameron afirma que el museo es un sistema de comunicación y, como tal, tiene implicaciones para la educación. Cameron, asegura que la estructura del museo es similar a la del medio de comunicación, aunque lo considera una institución más compleja al ser al mismo tiempo un emisor, medio y receptor. También considera que tiene muchas más ventajas sobre los media tradicionales al poseer la pieza, la obra de arte, el espécimen, que llama las “cosas verdaderas".

Estudiar la relación entre los museos y la inclusión social parte del convencimiento de que los museos y centros de ciencias han de potenciar la vertiente social que les corresponde como instituciones culturales, una vez consolidado su papel dentro de una sociedad que los identifica en 
sus funciones patrimoniales, educativas, y como mediadores entre el conocimiento científico y la sociedad.

No se trata de una temática nueva a nivel mundial, pero si se ha trabajado poco en nuestro país. De hecho, los museos británicos fueron los precursores en el planteamiento de esta relación que se investiga desde finales de los años 80 y se ha fortalecido a partir de la década de los 90 del siglo XX (Sandell: 2002), y extendido a los Estados Unidos y Australia.

En diferentes partes del mundo el tema de la inclusión de los públicos se ha venido trabajando de manera voluntariosa más que basada en una política de las instituciones, a pesar de que sean conscientes de su necesidad. Desde finales de los años 90 del siglo XX el debate ha dejado de pertenecer al ámbito teórico para moverse en el práctico (Sandell, 2002). Generalmente, la inclusión en los museos ha sido asumida desde el punto de vista de la accesibilidad física y psíquica; por ello, los programas y las acciones orientadas hacia colectivos como personas con discapacidades físicas y psíquicas se han emprendido desde hace más de dos décadas en diferentes partes del mundo.

Se ha elegido el público inmigrante teniendo en cuenta:

a. Que la tasa de residentes extranjeros en España ha crecido en los últimos 20 años alcanzando el 12\% d la población (Moreno \& Bruquetas, 2011: 11).

b. Que los museos en general, y los catalanes en particular, han trabajado con otro tipo de colectivos considerados vulnerables, especialmente los llamados públicos con diversidad funcional (física o psíquica).

Recordemos que el museo nace en la esfera privada, en sus inicios fue elitista y absolutamente excluyente. No obstante, los cambios sociales y políticos que ha vivido la humanidad han implicado transformaciones en todo tipo de instituciones y los museos son una de ellas. Sabemos que desde los años 60 del siglo XX el cambio del paradigma museístico ha sido fundamental en la relación que tiene hoy día la institución con el público. Su presencia, al igual que la de otros equipamientos culturales como las bibliotecas, ha adquirido protagonismo en la vida cotidiana de la ciudadanía. Gracias a los cambios sociales y políticos que la sociedad ha vivido, el museo pasó de la esfera privada a la pública abriéndose a todo tipo de personas.

En la actualidad, no se puede dudar del valor tanto patrimonial como educativo y comunicativo de los museos, ya que a lo largo de su historia se han consolidado y adaptado, en la medida de sus posibilidades, a un mundo más exigente y competitivo. La democratización de la cultura, arte y ciencia, a través de los museos públicos, y la divulgación del conocimiento científico son sólo dos de los aspectos positivos que han influido en el cambio de su relación con la sociedad y de la necesidad de su compromiso con los públicos vulnerables.

\subsection{La exclusión y la inclusión}

Atribuir a la exclusión el estatus de problema social tiene su origen en la Francia de la segunda posguerra mundial ligado al hecho de que el crecimiento en ese país fuera demasiado lento (Silver, 1994). El término se atribuye a René Lenoir, quien después fuera Secretario de Estado para la Acción Social del gobierno de Chirac. Lenoir agrupa a aquellas personas que no tienen ningún tipo de 


\section{Revista Latina de Comunicación Social \# 071 - Páginas 570 a 591 \\ Investigación | DOI: 10.4185/RLCS-2016-1110 | ISSN 1138-5820| Año 2016}

protección social, ni siquiera el cubrimiento de la salud, bajo el concepto de "excluidos". Este sector de la población entra en las políticas sociales de los 70 bajo los términos "integración" e "inserción" y tiene su fundamento teórico en los postulados de Rousseau respecto a la solidaridad, el nuevo contrato social y la cohesión (Silver, 1994).

Por ello, es importante que las instituciones no sólo prevean una inclusión intelectual, sino también tengan en cuenta que pueden promover la inclusión social y cultural (Fleming, 2013: 62-80; Sprünker, Munilla, Castellanos, 2014).

Hablar de exclusión es un tema recurrente no sólo en el mundo industrializado, sino especialmente sensible en los llamados países del "tercer mundo" que tienen que lidiar con problemáticas complejas. Países en los que más del $50 \%$ de la población vive en el lindar de la pobreza y en los que la llegada de la globalización y de la Sociedad del Conocimiento no han hecho más que incrementar las cifras de desigualdad especialmente en sectores tradicionalmente castigados como las minorías étnicas, los ancianos y los niños.

Es precisamente el concepto de ciudadanía el que se convierte en un punto clave en cuanto se considera una manera de contribuir a la inclusión. Para Marshall (1998) "a través de la relación entre la educación y la estructura ocupacional, la ciudadanía opera como un instrumento de estratificación social". Y en este sentido, los museos tienen mucho que decir como instituciones de educación no formal, uno de sus roles tradicionales.

El concepto de exclusión refleja también distintas nociones de integración social, solidaridad y ciudadanía que, además, se utilizan para el establecimiento de políticas sociales y culturales. Para Silver (2004), sin embargo, el establecimiento de categorías de personas excluidas puede contribuir a todo lo contrario e incluso, al surgimiento de guetos.

En el caso latinoamericano, uno de los investigadores que más ha trabajado el tema es Manfred MaxNeef quien promovió la teoría del Desarrollo a Escala Humana, en la que se superaba los modelos tanto del desarrollismo como del monetarismo tradicionalmente ligados a una posición economicista del desarrollo. Lo que propone Max-Neef y que han seguido investigadores como Antonio Elizalde (2002), entre otros, es basar el modelo en la satisfacción de las necesidades fundamentales del ser humano (Max-Neef, 2006).

En este modelo, la participación del individuo agrupado en las diferentes instancias de la sociedad civil, busca integrar a la población para superar el tradicional "rol paternalista del Estado Latinoamericano" (Max-Neef, 2006). El planteamiento de Max-Neef intenta, precisamente, dar relevancia a lo que llama "actores invisibles" que, en otras palabras, son los sectores poblacionales tradicionalmente excluidos y que pueden contribuir a establecer una sociedad más participativa a través del establecimiento de redes horizontales y de las micro organizaciones, estrategias que para el autor representan un aporte, más no la solución estricta de las desigualdades sociales.

Max-Neef, Silver, Elizalde, y otros autores destacan especialmente la exclusión socioeconómica. Elizalde (2002) llama la atención de un fenómeno particular y es el retroceso en el ascenso social entre una generación y otra. Se refiere a aquellos individuos pertenecientes a las clases más pobres, que han logrado completar sus estudios. En términos de Galbraith (1973) pertenecerían a la llamada "Nueva Clase" integrada por las personas con una amplia formación académica e intelectual. El 
problema es que el sistema no puede absorberlos y terminan cayendo de nuevo en la pobreza. En la misma situación se encontrarían muchos miembros de las clases medias cuya vida mejoró gracias al trabajo, el sacrificio y la dedicación, y como resultado de la crisis económica se ven desempleados, perdiendo sus casas y el nivel de vida que habían alcanzado. Situación extremadamente dura por cuanto es la primera vez que experimentan una crisis de tal relevancia (Galbraith, 1973). Es la situación que se vive actualmente en Europa y particularmente, en países como España en donde las generaciones mejor preparadas han tenido que emigrar buscando un lugar en el que puedan trabajar. Muchos de ellos pertenecientes a la clase obrera y a la clase media que no han tenido acceso a oportunidades laborales a pesar de su alta preparación.

\subsection{Museos e inclusión}

En el caso del patrimonio científico debemos tener en cuenta que el veloz desarrollo científicotecnológico genera desigualdades informativas entre aquellos que tienen conocimientos científicos y los que no. Los medios de comunicación especializados en ciencia y, entre ellos, los museos de ciencias se convierten en los "nuevos intermediarios culturales", que mencionaba Bourdieu (1988), pues su materia prima es la ciencia, que forma parte de los llamados "nuevos bienes". Estos "nuevos medios" se definen como aquellos que pertenecen al ámbito de la información y la educación (Bourdieu, 1988) y son consumidos por la "Nueva Clase". Galbraith y Bourdieu señalaban que la educación también se convierte en un medio de "distinción", de señalar diferencias entre los que han recibido formación y los que no. En este contexto, la información científica es un nuevo elemento para consumir y, de esta manera, acentuar las desigualdades entre aquellos que están informados y los que no.

En el actual escenario del consumo cultural enmarcado por los "nuevos intermediarios" y el consumo de los "nuevos bienes" que señala Bourdieu en la "Distinción" tenemos dos factores relacionados con el consumo de la información científica y tecnológica (Castellanos, 2010):

1.El consumo de la información no va necesariamente ligado al estatus social ni económico. Está relacionado con la percepción de que la ciencia y la tecnología es un asunto que compete sólo a los expertos.

2. La información sobre ciencia y tecnología es susceptible de convertirse en materia de desigualdad entre los que tienen conocimiento científico (aunque sea mínimo) y los que no. También entre los que pueden o no comprar tecnología.

En este contexto es necesario tener en cuenta que los estudios que tradicionalmente se han realizado en el tema de la Percepción Social de la Ciencia en la mayoría de países, no incluyen a la población rural, por lo cual, ya existe un marco diferenciador en el que sólo se tiene en cuenta la opinión urbana que, a la larga, es la que tiene un mayor acceso a la información, especialmente en los países en vías de desarrollo. En España, la institución encargada de realizar estos estudios es la Fundación Española para la Ciencia y Tecnología (FECyT), que realiza la encuesta de manera bianual desde 2002.

El abismo que se genera entre los grupos alfabetizados científicamente y los que no es susceptible de convertirse en causa de exclusión e, incluso, autoexclusión social, de la misma forma que lo es la 


\section{Revista Latina de Comunicación Social \# 071 - Páginas 570 a 591 \\ Investigación | DOI: 10.4185/RLCS-2016-1110 | ISSN 1138-5820| Año 2016}

ausencia de recursos económicos. Existen sectores sociales mucho más sensibles y que tradicionalmente viven en riesgo de marginación debido a su procedencia socioeconómica y, en algunos casos, geográfica.

A pesar de que algunos gestores piensan que los museos deben ceñirse a sus funciones más tradicionales, un gran número de museos no ignora el entorno en el que está ni la situación en la que vivimos. Estamos inmersos en diferentes crisis (económica, política, social, de seguridad, de valores, etc.) la sociedad de hoy debe afrontar un cúmulo de situaciones que le envuelve en la incertidumbre (Beck, 1992) y la sensación de riesgo. En este contexto, no sólo el trabajo de las instituciones es fundamental, sino que también se requieren esfuerzos gubernamentales de fondo para establecer políticas que redunden en esta función del museo. Como señala Sandell (2003), para que los museos se conviertan en agentes eficaces para la inclusión (Dawson, E., 2014a, 2014b) es necesario un cambio de paradigma en la función social del museo y de sus prácticas de trabajo.

A pesar de la tradición de otros museos europeos y latinoamericanos en la atención a la población en riesgo de exclusión, los programas estables en España son escasos, así como el estudio de sus resultados. En muchas ocasiones se continúa ligando los estudios sociales a los de educación y didáctica del museo, lo que no permite el avance de esta línea. Esta situación puede deberse a que España es un país que es receptor de inmigrantes desde hace poco, en comparación con otros como Estados Unidos y el Reino Unido.

La experiencia del Reino Unido muestra dos líneas claras que se derivan de todo lo que hemos visto anteriormente:

1. La línea centrada en atender a los públicos con discapacidades físicas y/o psíquicas

2. La línea centrada en aquellas personas afectadas por otro tipo de exclusiones (sociales, económicas, víctimas de racismo, inmigrantes, etc.)

Estas líneas se han visto impulsadas $\mathrm{y}$, de alguna manera, favorecidas por las políticas gubernamentales tanto sociales como culturales que permitieron, por ejemplo, que en el año 2000 se publicara uno de los estudios más completos en la relación "Museos e inclusión social" elaborado por el Group of Large Local Authority Museums (GLLAM) en el que participaron 22 museos y galerías de todo el país (Castellanos, 2013).

En los Estados Unidos, una de las experiencias más recientes es "Cultures Connect All. Rethinking Audiences in Times of Demographic Change" realizada en 2011, en el que diferentes museos intercambiaban experiencias relacionadas con la atención a los miembros de las comunidades más frágiles. Lo importante de esta experiencia fue el reconocimiento de la necesidad de contribuir a la mejora en la vida de dos grupos poblacionales clave, los mayores de 65 y los inmigrantes, teniendo en cuenta las necesidades de cada uno en todos los ámbitos de su existencia: desde la salud, la educación y el manejo de las finanzas, hasta el aislamiento que viven muchas personas de estos grupos. La importancia de trabajar con estos dos colectivos viene dada porque se calcula que en los Estados Unidos, en los próximos 20 años, los mayores de 65 serán más de 70 millones. Además, los inmigrantes que llegaron entre 2000 y 2007 fueron más de 10 millones de personas. De este colectivo, el 20\% tiene 55 años o más (Partners for Livable Communities, 2011: 13). 
A 30 junio de 2013, se contabilizaron en España 5.503. 977 extranjeros con certificado de registro o con tarjeta de residencia. A pesar de la crisis que vive el país, se produjo un aumento del $1,70 \%$ respecto al mismo período del año anterior, según las cifras del Observatorio Permanente de la Inmigración (2013: 1). En Cataluña viven 1.261.416 inmigrantes, incluidos los residentes comunitarios, lo que representa el 22,9\% (Ministerio de Empleo y Seguridad Social: 2013). Cómo señalan Moreno y Bruqueta (2011), a pesar de la crisis, el número de inmigrantes en España es alto y por ello es necesario que también las instituciones culturales tengan en cuenta a este colectivo, tal y cómo se ha visto en el ejemplo de los Estados Unidos (Partners for Livable Communities, 2011).

\subsection{Museo e identidad}

Para esta investigación preliminar, no se pretende ahondar en los aspectos relacionados con la identidad. Sin embargo, se indaga sobre las contribuciones del museo (a través de los elementos patrimoniales comunes y distintos) como parte de las instituciones culturales del país de acogida.

En primer lugar, y antes de situar la identidad en el interior de un museo, debemos tener en cuenta que muchas son las teorías y teóricos que han disertado y trabajado ampliamente sobre el tema. Y, en Europa, es un tema actual en el cual se está trabajando para su comprensión conceptual y contextual (Galla, Amareswar en Braendholt Lundgaard, Ida y Thorek Jensen, Jacob, 213: 64).

Para este primer trabajo, nos centramos en la noción de identidad desde la perspectiva de los Cultural Studies. Se trata de un concepto central para esta corriente teórica presente en la mayoría de sus investigaciones: desde los análisis sobre la cultura popular, hasta los estudios de género. La identidad tiene que ver con la manera como se ve a sí misma una persona (Barker, 2001): los Cultural Studies se preocupan por conocer cómo el individuo llega a ser como es, cómo produce como sujeto, y cómo se describe a sí mismo (mujer, hombre, negro, blanco, indígena, panameño, etc.).

La visión de la identidad como producto de una herencia común, de unos ancestros comunes y unos recursos simbólicos compartidos, la denomina Stuart Hall "posición esencialista" y desde este punto de vista es posible hablar de la "identidad británica", por ejemplo, (Barker, 2001: 176). Sin embargo, Hall defiende también la posición "anti-esencialista" de la identidad, es decir, organizada alrededor de los puntos de diferencia. Para Hall es importante tener en cuenta que la identidad no es un estado natural o algo dado por sentado, sino un proceso en construcción basada tanto en las similitudes como en las diferencias originadas, especialmente, por la multiculturalidad de las sociedades contemporáneas. Y es entonces, cuando recomienda trabajar con base en las "articulaciones", la unidad de dos elementos diferentes, para poder trabajar el tema de la identidad. En este mismo sentido, García Canclini (1997) se refiere a las intersecciones como el lugar en el que "las narrativas se oponen o se cruzan". García Canclini (1997) utiliza el término hibridación, que abarca "diversas mezclas interculturales" y va más allá del concepto de sincretismo. En el mundo anglosajón, a finales de los años 80 se acuña el concepto de "glocal" asociado a los procesos capitalistas en los cuales se localiza lo global y se globaliza lo local (Escobar, 2001) y que responde a la propuesta de Latour de hibridar los dos términos (Prazniak \& Dirlik, 2001: 28).

Estas posiciones planteadas en la década de los 90 del siglo XX son aplicables a las sociedades de hoy en las que los procesos generados por la globalización y los movimientos migratorios han 


\section{Revista Latina de Comunicación Social \# 071 - Páginas 570 a 591 \\ Investigación | DOI: 10.4185/RLCS-2016-1110 | ISSN 1138-5820| Año 2016}

desplazado el concepto de identidad. Literalmente, lo han movido de lugar. Las fronteras y el territorio se muestran cada vez más difusos y el desarrollo de las TIC, especialmente el crecimiento de las redes sociales, contribuyen a ello.

Es en este contexto en el que la construcción de la identidad desde la perspectiva de Rounds (2006) es pertinente. Construir la identidad requiere mantener y adaptar nuestra identidad y persuadir a otros a creer en ella. Se trata de una construcción fundamental para esta sociedad globalizada en la que las fronteras se difuminan. Siguiendo a Jay Rounds (2006), no se trata de una identidad marcada exclusivamente por el lugar de nacimiento, sino relacionada con el lugar y las maneras cómo se construye la vida, coincidiendo con los teóricos de la Cultural Studies, tanto anglosajones como latinoamericanos. Este aspecto es fundamental en sociedades marcadas por los procesos migratorios. Hablar de identidad no implica cerrarse al mundo, significa también invitar al otro a que te conozca y te reconozca.

Volviendo al concepto de identidad en los museos, se trata de un tema inherente al nacimiento de este equipamiento. Las grandes instituciones museísticas nutren sus colecciones de objetos que simbolizan la posición esencialista de Hall a la que nos referíamos anteriormente (el arraigo, la tradición, lo común, lo heredado) y, en las sociedades multiculturales, reflejan la posición antiesencialista (las diferencias) definida por el investigador británico.

Se trata de que los MCYT consigan que dialoguen las diferentes identidades que coexisten en cada comunidad. El único modelo posible es el que cada uno diseñe según su contexto y realidad.

La interacción o el diálogo entre las diversas identidades y el museo puede expresarse gráficamente de la siguiente manera:

Gráfico 1. Identidad y museo.

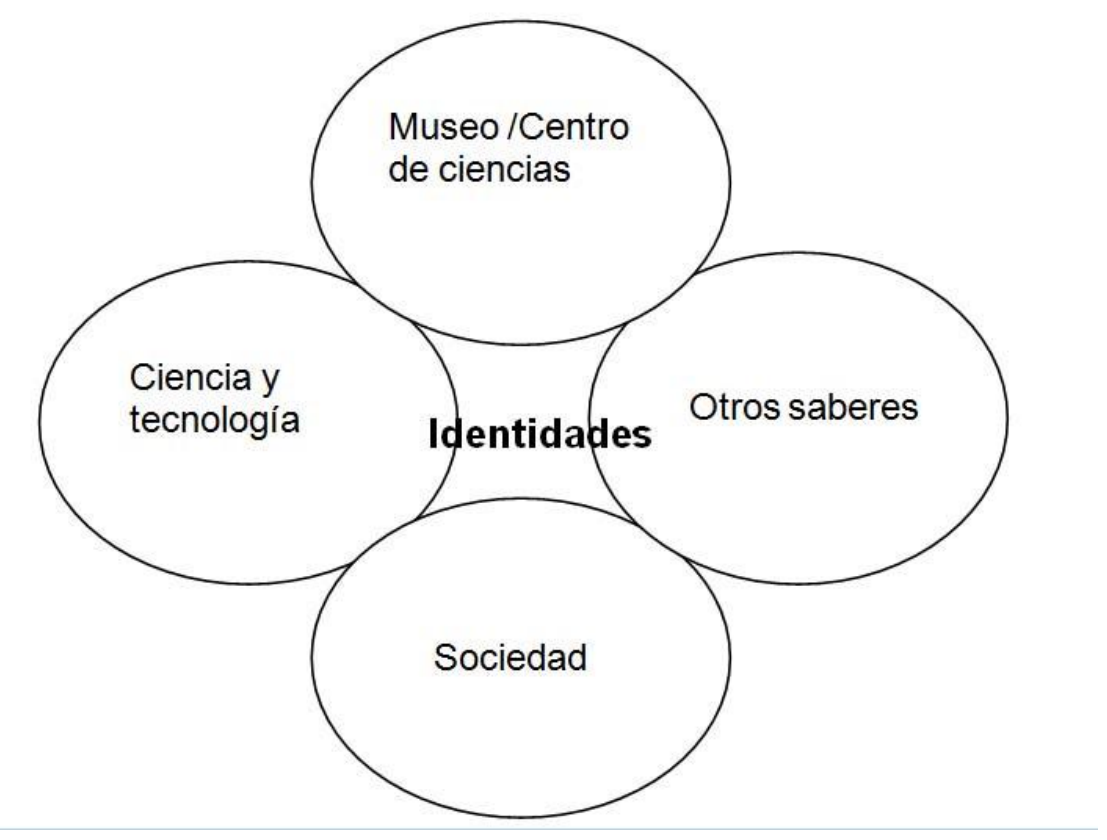

Fuente: Castellanos (2011) 
Como se observa en el gráfico, el museo no es sólo un mediador, sino también el lugar en el que se encuentran y desarrollan las diferentes identidades de un individuo, teniendo en cuenta que el patrimonio alberga y proporciona conocimientos muy concretos y propios que podemos adquirir y pueden formar parte de nuestra identidad personal o colectiva (Sprünker, 2011). Este proceso, puede contribuir a afianzar la relación ciencia-sociedad.

Al relacionar el tema científico con un inmigrante, no queremos mirar su bagaje académico, o qué aprendió en la visita a una exposición, buscamos explorar cómo perciben su identidad al entrar al museo: ¿hay objetos que le ayudan a entender a los nativos de la ciudad que habitan?, ¿alguna pieza o espacio les recuerda su lugar de origen? Insistimos en que en este estudio preliminar la identidad se asume como un tema exploratorio.

\section{Método}

\subsection{Participantes}

Los museos con los que se ha iniciado la investigación son dos:

El Cosmocaixa es un museo de ciencias perteneciente a la Obra Social de "la Caixa" situado en Barcelona. Fue inaugurado en el año 2004 tras la remodelación de su predecesor, el Museo de Ciencias de Barcelona, inaugurado en 1981. Tiene un gran protagonismo en la vida científica de la ciudad. Es sede habitual de actividades de divulgación científica que van más allá de sus funciones patrimoniales y educativas. La institución ha sido una de las inspiraciones de muchos centros de ciencias y museos de ciencias del mundo, especialmente de España y América Latina. La Fundación "laCaixa" tiene uno de los programas más importantes de España a nivel social. En 2012 dedicó a los programas sociales más de 200 millones de euros (Obra Social "la Caixa": 2012) https://obrasocial.lacaixa.es

Y el Museo Marítimo de Barcelona es uno de los pioneros en España en el trabajo con personas en riesgo de exclusión socio económica. Se encuentra ubicado en el Raval, uno de los barrios más antiguos y con algunos de los mayores índices de pobreza de Barcelona, además de ser uno de los sectores con mayor asentamiento de inmigrantes, especialmente, paquistaníes y marroquíes. Es el primer museo español (y el segundo europeo) en tener un departamento de Responsabilidad Social y parte del hecho de que el acceso a la cultura es uno de los derechos fundamentales de los ciudadanos. El edificio de la institución, recientemente reformado, goza de una situación inmejorable en el que se han derribado las barreras arquitectónicas. El Museo está en el edifico de las Atarazanas Reales, en la fachada marítima de la ciudad y al pie de la montaña de Montjuïc, Monumento Histórico-Artístico desde 1976, y de gran relevancia histórica en la construcción de barcos. En 2006 fue declarado Museo de Interés Nacional

(http://www.mmb.cat/informacio_general.php?idm=2\&pagina=1\&estic=0). La gestión es del Consorci de les Drassanes Reials i Museu Marítim de Barcelona, formado por el Ayuntamiento de Barcelona, la Diputació de Barcelona y la Autoridad Portuaria de Barcelona.

En estos momentos trabaja con la Universidad Politécnica de Catalunya (UPC) a fin de desarrollar un software especial para personas ciegas o con visión reducida. En 2011, en compañía de la cooperativa Norai - Raval SCCL, Impulsem SCCL y la Fundació Surt, establecieron el proyecto NORAI mediante el cual buscaban, entre otras cosas, contribuir a la mejora de la calidad de vida de 
los habitantes del barrio. Para ello, NORAI tiene la doble función de tener en cuenta la gastronomía marítima y contribuir en la formación y la inserción laboral de las personas en riesgo de exclusión social del entorno, especialmente inmigrantes. En la presente investigación se cuenta con la colaboración de la Asociación Diàlegs de dona, que trabaja con mujeres inmigrantes procedentes de países extracomunitarios, para facilitar su integración en la sociedad catalana.

\subsection{Estrategias metodológicas}

Para poder alcanzar los objetivos propuestos en el estudio preliminar, se utilizó una técnica cuantitativa de auto-informe (la encuesta) y dos cualitativas (la observación no participante y el análisis documental). Los datos se recopilaron a través de encuestas con preguntas abiertas y cerradas, y se incluyó una escala no verbal.

Las encuestas permiten obtener información de primera mano por parte de los encuestados. Es una de las técnicas de auto-informe más utilizada en las instituciones patrimoniales.

Respecto a las encuestas, en el caso de "Cosmocaixa" fueron autogestionadas y en el Museo Marítimo fueron presenciales, post-visita y realizadas en la Asociación Diàlegs de Dona, con preguntas abiertas y cerradas. La encuesta se conformó para recoger información sobre tres bloques:

a) Relación del encuestado con el museo

b) Relación museo-identidad

c) Perfil sociodemográfico

Se introdujo una escala no verbal para que el visitante externalizara a través de un ícono (:), :,$: 1)$ sobre cómo percibía el lugar de la visita. De esta manera se obtuvo un elemento emocional para identificar si el inmigrante se sentía cómodo en el museo, si sentía la emoción contraria, o si le era indiferente.

El modelo de encuesta se ajustó después de analizar los primeros datos recogidos en el Cosmocaixa, para cambiar aquellas preguntas que no proporcionaron información. También, se realizaron cambios a solicitud de la asociación que colaboró en la visita en el Museo Marítimo, pues la mayoría de las mujeres que participan en sus actividades son analfabetas o no conocen ninguno de los dos idiomas (castellano o catalán).

La muestra obtenida en este estudio preliminar es pequeña, pero se debe tener en cuenta que además de ser un estudio previo, McClafferty y otros (1996: 2 y ss.), resaltan que en los museos las muestras pequeñas pueden ser suficientes para encontrar indicios y que se pueden interpretar con sentido común más que con complicados métodos estadísticos.

\subsection{Procedimiento}

Se realizó una prueba en cada uno de los museos de la siguiente manera: 
En el Cosmocaixa se realizó una prueba piloto para comprobar las preguntas de la encuesta, durante la primera quincena de julio de 2013, en la que se recogen 19 encuestas de público inmigrante elegido al azar, que iba a Cosmocaixa a realizar una visita. De estas 19 se invalidan tres por haber sido respondidas en grupo. No se diferenciaron las visitas realizadas en días laborables y las de fin de semana.

Imagen 1. Exposición y visitantes en el Cosmocaixa.

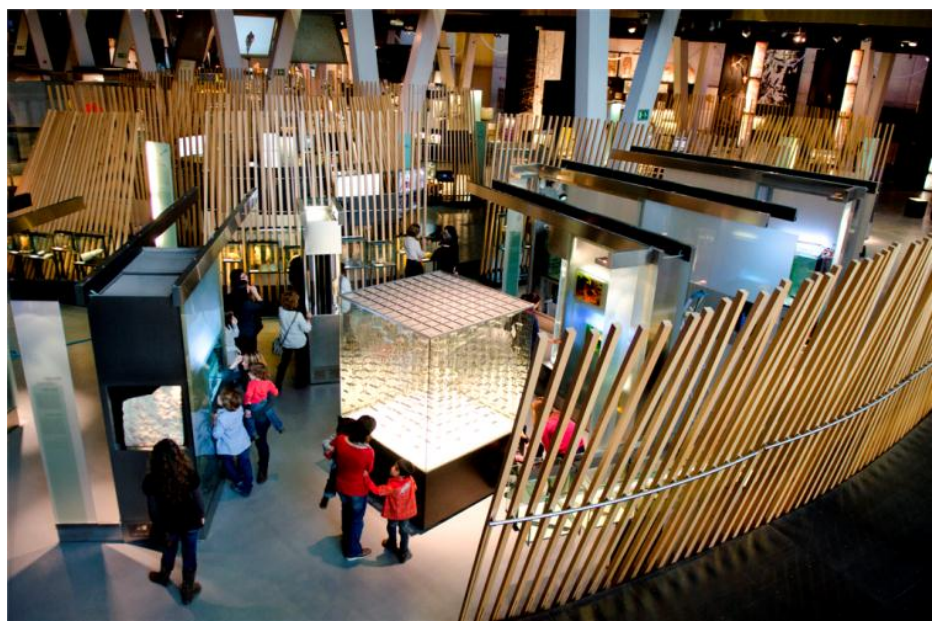

Fuente: Cosmocaixa. Escarlata Blanco.

Y en el Museo Marítimo se recogieron los datos el día 14 de febrero 2013. Posteriormente se recopilaron 20 encuestas de público inmigrante. Para realizar el estudio, el Museo programó una visita diseñada a medida para un grupo de mujeres que visitan la asociación "Diàlegs de dóna" durante una hora y media a lo largo de la mañana. Se procedió de la siguiente forma:

\section{Imagen 2. Grupo de mujeres participantes en la visita en el Museo Marítimo.}

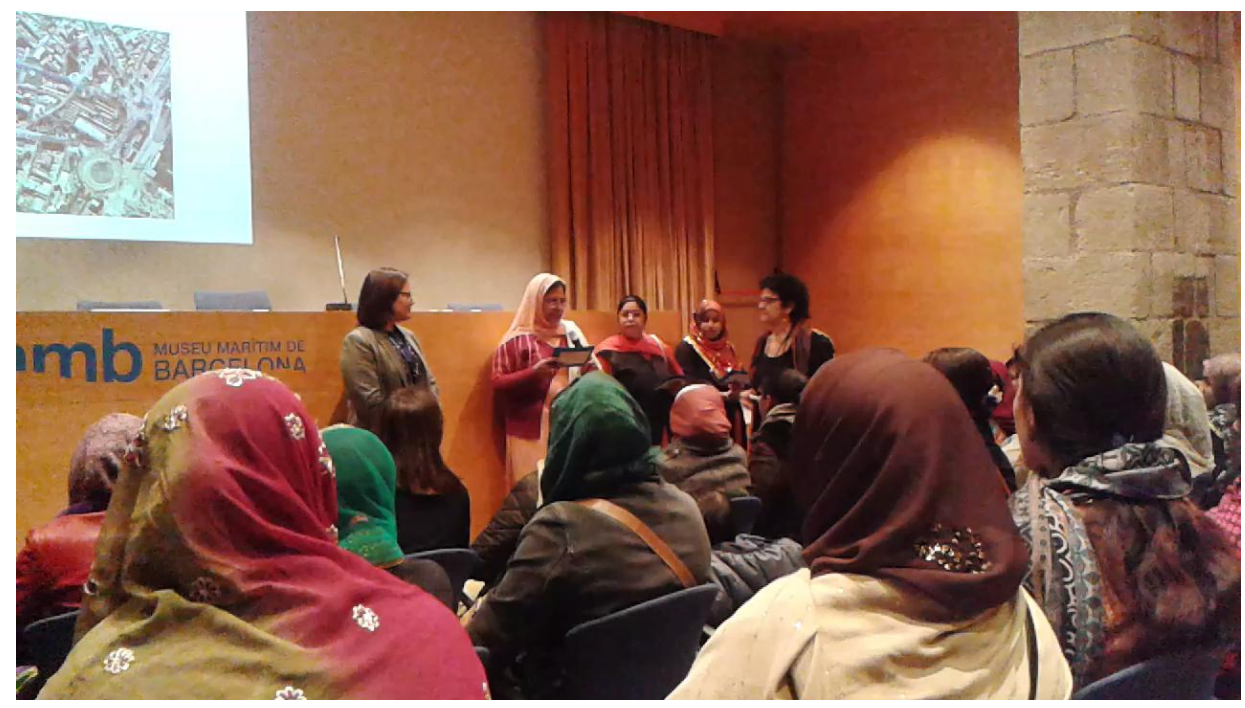

Fuente: Visita Museo Marítimo con mujeres inmigrantes. Patricia Castellanos (2013). 


\section{Revista Latina de Comunicación Social \# 071 - Páginas 570 a 591

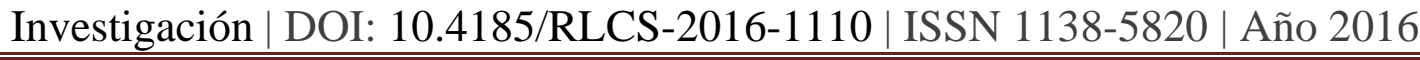

a. La coordinadora de Responsabilidad Social del Museo, Teresa Soldevila, hizo la presentación del museo en la que se explicó a las asistentes las características del edificio histórico en el que se encuentra y a qué se dedica la institución. También se explicó qué es el patrimonio cultural. La presentación tuvo que acortarse por la necesidad de hacer la traducción. Hay que recordar que las mujeres participantes son recién llegadas o no conocen el idioma, y por esta razón fue necesario traducir previamente el guión de la funcionaria del museo a los idiomas de las asistentes. Este trabajo lo realizaron las educadoras de la asociación "Diàlegs de dona".

b. Se realizó la visita con 43 mujeres procedentes de Pakistán, India y Bangladesh, de las cuales 20 respondieron la encuesta post-visita. Para ello, fue necesario traducir previamente el guión de la funcionaria del museo a los idiomas de las asistentes: Urdu, Indi y Bangla. Este trabajo lo realizaron las educadoras de la asociación con algunas de las participantes que entendían el castellano o que hablaban inglés. La dinámica de la visita fue intercalar la presentación de Teresa Soldevila, con la traducción de tres mujeres que hablan inglés o castellano. Durante la actividad, se realizó una observación no participante para ver las reacciones de las mujeres ante la visita

c. Una semana después de la visita la ONG aplicó la encuesta con la misma dificultad del idioma. La herramienta debió simplificarse al máximo para facilitar la toma de datos.

Una vez recogidos los datos e informaciones, se procedió al análisis. Concretamente, se realizó un análisis de contenido categorizando los diferentes adjetivos de las respuestas.

\section{Resultados}

Al tratarse de una investigación preliminar, los resultados deben interpretarse teniendo en cuenta esta circunstancia.

\subsection{Cosmocaixa}

Respecto a la encuesta a inmigrante elegido al azar:

a) Bloque 1. Relación con el museo

La relación de los inmigrantes encuestados con Cosmocaixa, no es nueva: de los 16 encuestados, 14 habían visitado el museo, mientras que sólo 2 hacían una primera visita. Al ser preguntados sobre el hecho de no haber visitado antes el museo, los encuestados dijeron que no sabían dónde estaba y por razones laborales.

\section{Gráfico 2. ¿Ha visitado alguna vez el museo?}

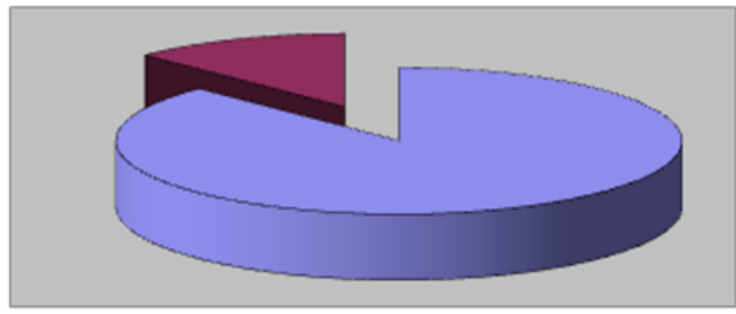

口Ha visitado museo antes

口No lo ha visitado

Fuente: elaboración propia 


\section{Revista Latina de Comunicación Social \# 071 - Páginas 570 a 591 Investigación | DOI: 10.4185/RLCS-2016-1110 | ISSN 1138-5820| Año 2016}

En cuanto a la revisita, seis habían ido entre 1 y 3 veces; y nueve, habían ido más de tres veces a la institución.

Al ser cuestionados mediante una escala no verbal sobre lo que pensaban del museo, todos los participantes eligieron la escala más positiva $:$. Esta buena opinión sobre la institución se ve reflejada por el hecho de que 14 encuestados aseguraban sentirse cómodos dentro del Cosmocaixa debido al espacio, los contenidos, la limpieza del lugar, la temperatura, y la señalización.

Cuando se trata de realizar una segunda visita, el factor tiempo no influye en la decisión. Siete de los encuestados afirmaron no estar de acuerdo con que la falta de tiempo influya en su visita.

En cuanto a la afirmación "Prefiero otra actividad", dos encuestados se mostraron indecisos, dos estaban poco de acuerdo y siete, nada de acuerdo. Once de los participantes volverían con alguien. Preguntados sobre el interés por el museo, 9 personas no estaban de acuerdo con que el museo no les interesa, y dos estaban poco de acuerdo. Tres personas estaban poco de acuerdo en que el Cosmocaixa no era para ellos y ocho no estaban nada de acuerdo.

Interesados en conocer si el museo es un elemento que contribuya a integrar al inmigrante en la sociedad, se pregunta sobre si la visita a Cosmocaixa les ha hecho sentirse parte del país. Cinco personas estaban muy de acuerdo, cinco participantes estaban bastante de acuerdo, un indeciso, uno que no estaba de acuerdo, y 4 que no respondieron.

\section{Gráfico 3. ¿La visita a Cosmocaixa les ha hecho sentirse parte del país de acogida?}
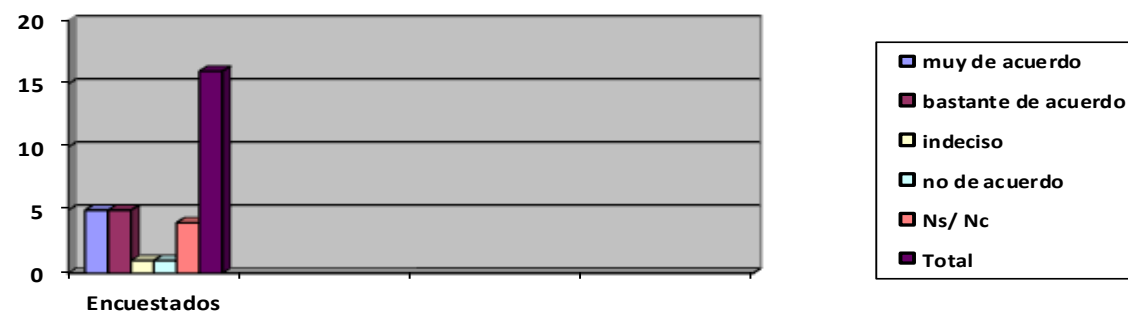

Fuente: elaboración propia

b)Bloque 2. Relación museo- identidad

c)

Este bloque quiere revisar si el visitante ve alguna relación entre el Cosmocaixa y su identidad.

Uno de nuestros puntos de partida es la identidad en el sentido de Jay Rounds (2006): no se trata de una identidad marcada exclusivamente por el lugar de nacimiento, sino relacionada con el lugar y el modo en que se construye la vida. Teniendo en cuenta esta consideración, preguntamos si el Cosmocaixa hace sentir al visitante más cercano al lugar de acogida y al lugar de origen. Así pues, siete encuestados se sintieron más cercanos a Barcelona y sus vecinos de aquí; seis participantes afirmaron sentirse más cercanos a España, a su país de origen, a la familia, a los amigos que no viven aquí y a sus amigos de aquí. Para cuatro personas, la institución les hizo sentir más cercanos al país de sus padres. 
Gráfico 4. ¿El Cosmocaixa hace sentir al visitante más cercano al lugar de acogida y al lugar de origen?
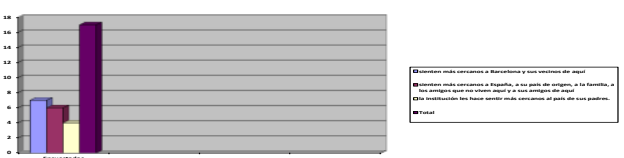

Fuente: elaboración propia

Intentando averiguar la relación entre la identidad propia y el Cosmocaixa, se planteó la siguiente pregunta: “¿Hay algún elemento que te haga recordar tu lugar de origen? (ten en cuenta los elementos expuestos, los conceptos trabajados, el ambiente, la arquitectura, y otros que quieras mencionar"). Diez personas consideraron que sí y señalaron algún elemento del museo (siete nombraron el "Bosque inundado" [2]. Los otros seis consideraron que no había nada que les recordase su lugar de origen.

\section{Gráfico 5. ¿Hay algún elemento que te recuerda a tu lugar de origen?}

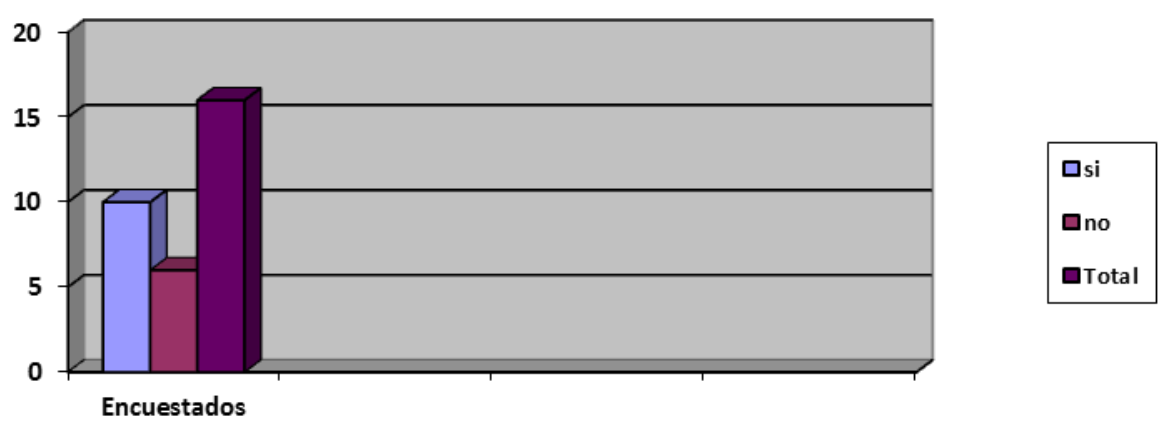

Fuente: elaboración propia

A la pregunta contraria, "¿Hay algún elemento muy diferente a tu lugar de origen que te haya llamado la atención?", la mitad de participantes afirmaron que no y la otra mitad que sí, pero no especificaron qué elementos encontraban diferentes.

d)Bloque 3. Perfil socio-demográfico

La encuesta fue respondida por 10 mujeres y seis hombres $(37,5 \%)$. Once de ellos se situaban entre los 29 y los 49 años. Dos entre los 18 y los 28 años. Uno entre los 50 y los 60, y dos superaban los 60 años. 


\section{Revista Latina de Comunicación Social \# 071 - Páginas 570 a 591 \\ Investigación | DOI: 10.4185/RLCS-2016-1110 | ISSN 1138-5820| Año 2016}

\section{Gráfico 6. El género de los visitantes y encuestados}

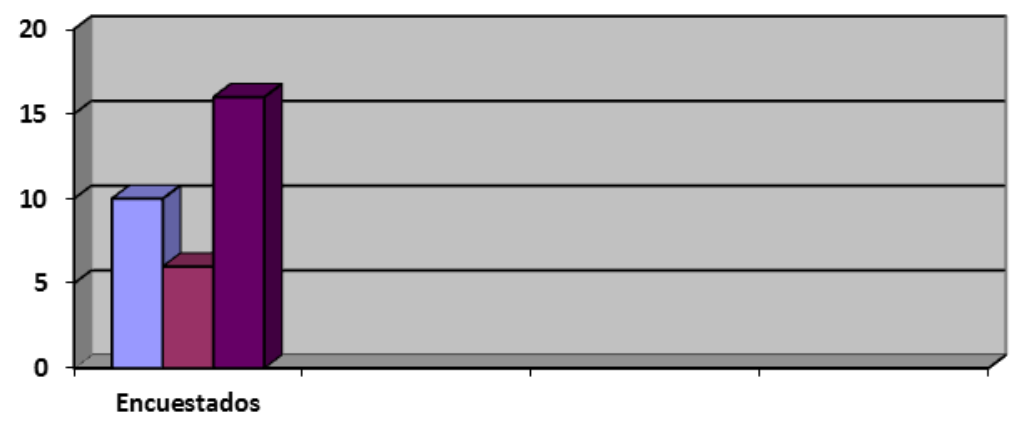

Fuente: elaboración propia

En cuanto al lugar de nacimiento, tres de los participantes en la muestra de la prueba piloto nacieron en Italia. Los otros participantes provienen de otros países: Alemania, Brasil, China, Colombia, Cuba, Marruecos, México, Perú, Polonia, República Dominicana, Uruguay y Venezuela.

En cuanto a los años de residencia en Cataluña dos personas llevan entre 1 y 3 años; nueve personas viven aquí entre 4 y 10 años, y 6 llevan más de 10 años residiendo en Barcelona.

La ocupación actual es diversa: ama de casa, investigador, madre, empresaria del hogar, traductora, dependienta, cocinera, profesora instituto, administrativo, auxiliar de geriatría, dos estudiantes y dos jubilados. De la misma forma, las profesiones son variadas: diseñadora industrial, informático, biólogo, estudiante, limpieza, director de empresas, dentista, ama de casa, bellas artes, cocinera, profesora de instituto, y sanitario.

Los estudios máximos alcanzados son universitarios en el caso de siete personas, cuatro han cursado el bachillerato, una tiene un grado medio y uno más una diplomatura. Uno tiene un posgrado y dos tienen máster.

\section{Gráfico 7. Nivel de estudios}

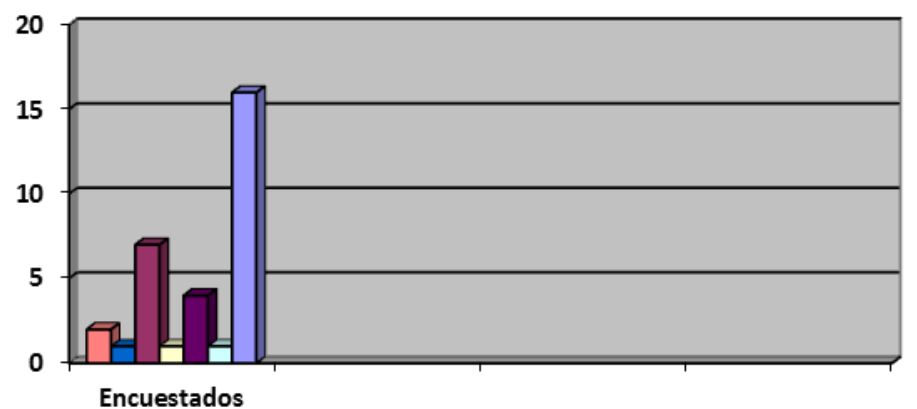

पMáster
口Posgrado
口Estudios universitarios
$\square$ Diplomatura
$\square$ Bachillerato
$\square$ Grado medio
$\square$ Total

Fuente: elaboración propia 


\section{Revista Latina de Comunicación Social \# 071 - Páginas 570 a 591 Investigación | DOI: 10.4185/RLCS-2016-1110 | ISSN 1138-5820| Año 2016}

\subsection{Museo Marítimo}

Respecto a la encuesta realizada después de la visita comentada diseñada a medida para un grupo de mujeres inmigrantes que visitan la asociación:

a) Bloque 1. Relación con el museo

De las 20 mujeres que contestaron la encuesta, de un total de 43 que realizaron la visita, 12 van al museo por primera vez. De las que no habían ido nunca al museo, la mitad aseguró no conocerlo. Cinco encuestadas aseguraron haber ido al museo más de tres veces y tres de ellas dijeron haber oído hablar de él a sus maridos. De las participantes, 13 indican que les gusta y que es fácil llegar. A 11 les parece que les conecta con el país de acogida.

Gráfico 8. Para la mayoría de las encuestadas, el museo Maritim, es el primer museo que visitan

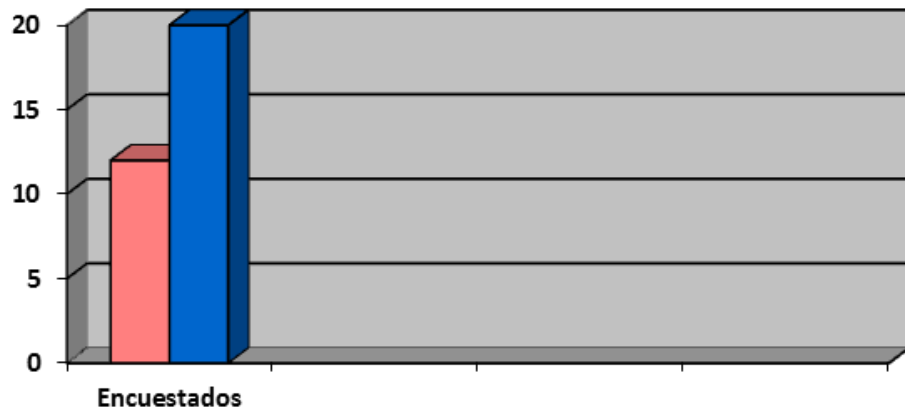

口Primera vez en el MM

aTotal

Fuente: elaboración propia

b) Bloque 2. Relación museo- identidad

De las encuestadas, 16 indican que encuentran elementos que les recordaron su país de origen; pero 11 consideraron que hay elementos muy diferentes a su país, aunque no señalaron de manera concreta cuáles. Algunas mencionaron el hecho de que los barcos expuestos o son más grandes o son más pequeños que los que ven en su lugar de nacimiento.

\section{Gráfico 9. ¿Hay algún elemento que te recuerda a tu lugar de origen?}
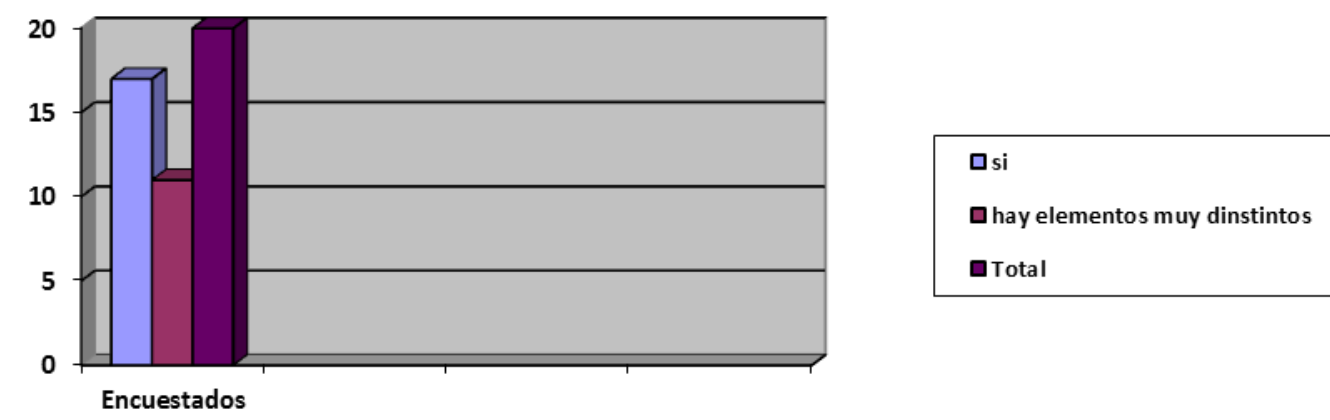

Fuente: elaboración propia 


\section{Revista Latina de Comunicación Social \# 071 - Páginas 570 a 591 \\ Investigación | DOI: 10.4185/RLCS-2016-1110 | ISSN 1138-5820| Año 2016}

Para 10 participantes, la visita al museo no les ayudó a entender más a Cataluña, y el mismo número consideró que tampoco le ayudó a entender a España. Pero para 8 mujeres, la visita ha contribuido a entender más a sus amigos de Barcelona y a 5 les ha ayudado a entender más su país, opinión contraria a la de 6 encuestadas.

\section{Gráfico 10. ¿El Museo Marítimo les ha ayudado a entender más el país de acogida?}
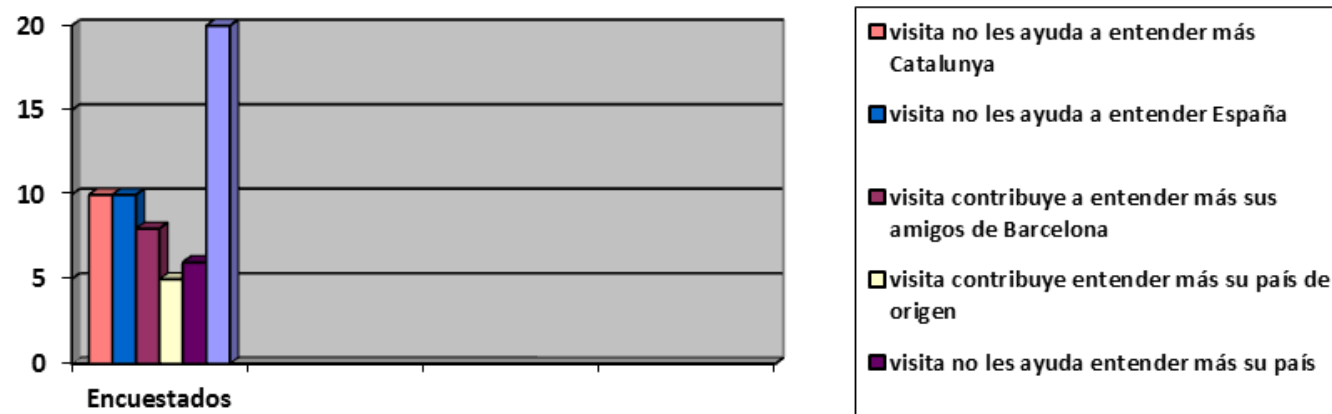

Fuente: elaboración propia

c) Bloque 3. Perfil socio-demográfico

Las mujeres encuestadas son jóvenes: 6 se encuentran entre los 15 y los 17 años; 5 entre los 26 y los 30; 3 entre los 31 y los 35; 2 entre los 36 y los 40, y sólo una supera los 41 años, 3 que no respondieron. En cuanto a los países de origen, 10 son de Bangladesh; 2 de India; 2 de Pakistán; 1 de Indonesia y una de Nepal. Las 4 restantes no respondieron la pregunta. En cuanto al tiempo que llevan viviendo en Barcelona, 12 llevan menos de cinco años; 1 entre 5 y 10 años; 3 llevan más de 10 años, y 4 no lo reportan.

\section{Gráfico 11. Edades.}

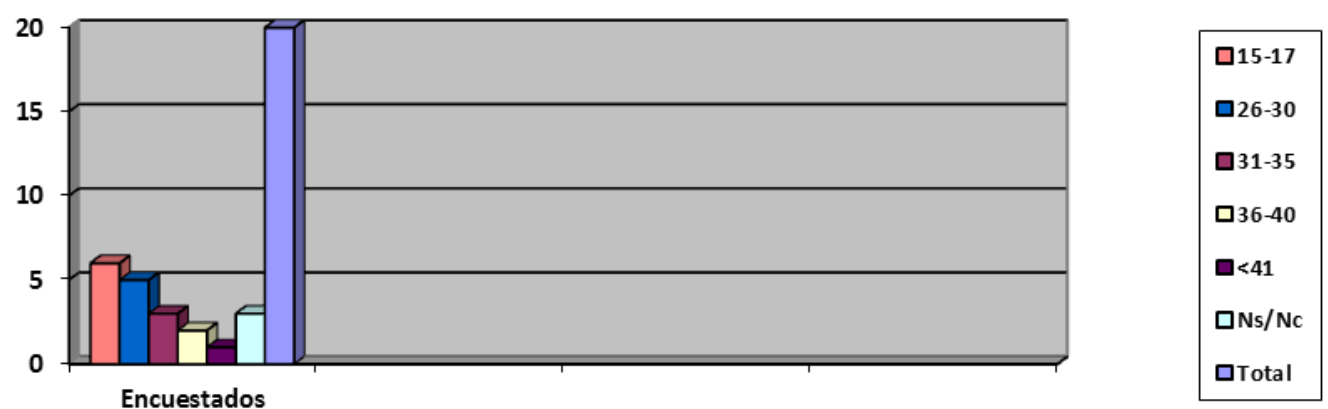

Fuente: elaboración propia

Respecto a la ocupación, ninguna de las 20 participantes trabaja. Y en lo referente a los estudios, 2 manifiestan tener estudios universitarios; una tiene estudios técnicos, 5 tienen la secundaria; y 5 más tienen estudios primarios. 7 mujeres no respondieron. 


\section{Gráfico 12. Nivel de estudios}

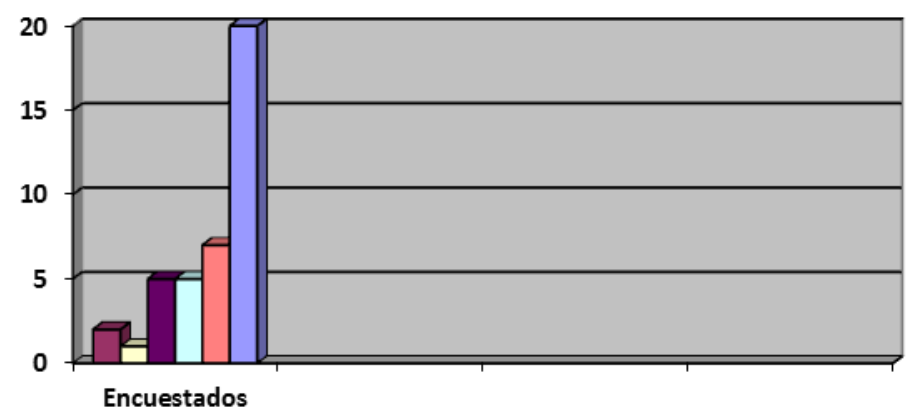

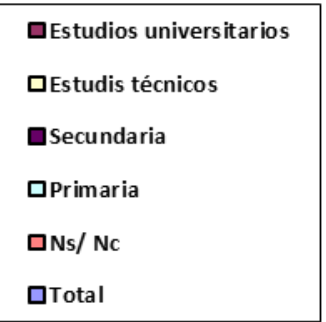

Fuente: elaboración propia

Respecto a la observación no participante. Se recogieron las actitudes de las participantes observadas durante la visita.

a. Charla previa a la visita a la sala: Al principio, las mujeres se mostraron serias y calladas cuando entraron en el auditorio. Su actitud fue cambiando cuando el grupo estuvo completo. Al escuchar la traducción en su idioma, cada vez que la encargada leía la traducción de la funcionaria, se mostraron alegres y aplaudieron. Un aspecto que les gustó fue el hecho de que el museo es gratis los domingos.

b. Visita a sala: Las asistentes se mostraron impresionadas al entrar en la sala. Miraban las dimensiones y los techos altos y señalaban los diferentes barcos. Tomaron fotos de ellas, de las profesoras y de los barcos que les gustaban. Algunas comentaron, que algunos de los barcos expuestos se parecían a alguno que había en su país. En estos casos se mostraron sorprendidas. Durante la visita vieron algunos grupos escolares y comentaron que el domingo llevarían a sus familias y a sus niños. Al finalizar, tomaron una foto del grupo y se mostraron alegres con la visita. Una comentó que su hijo había estado en el museo en una visita escolar.

\section{Discusión y conclusiones}

En el caso de España el trabajo para identificar la relación entre los MCyT y el público inmigrante es reciente. El crecimiento de la población foránea en la última década ha cambiado el panorama poblacional del país por lo que se hace necesario tener en cuenta a las personas de fuera, dentro del llamado "Nuevo público" que buscan las instituciones culturales.

Por eso, cuando se habla de inclusión social y de lo excluyente que puede llegar a ser el acceso a la información científica es necesario tener en cuenta que, de momento, sólo se ve la punta del iceberg, es el comienzo de un análisis que por ahora cubre a la población urbana. Establecer estrategias que permitan que la información científica llegue a todas las capas de la sociedad, tiene que pensarse de manera similar a como se busca la penetración de las TIC. Más allá de crear salas de exposiciones itinerantes, por ejemplo, se necesita tener en cuenta que la accesibilidad no es únicamente física sino sobre todo de igualdad de oportunidades, de acceso al conocimiento y la visita a medida del museo 
Marítimo o los interactivos de Cosmocaixa son un modo de hacer accesible el conocimiento al público inmigrante.

Aunque las experiencias presentadas son apenas una pequeña muestra de futuros estudios, sí dan alguna pista de la relación de la comunidad inmigrante con los MCyT. Por ejemplo, hay indicios de que son un lugar que contribuye a la integración en la sociedad de acogida, a comprender el país al que han llegado así como a sus habitantes. Otro aspecto a tener en cuenta es que, en el caso del Cosmocaixa, el perfil nos habla de inmigrantes que en su mayoría tiene un alto nivel de estudios, lo que corresponde al visitante medio de los MCyT, y que revisita el museo.

La experiencia en el Museo Marítimo fue completamente diferente, teniendo en cuenta que se trató de una visita ex professo, pero igual de válida para sondear a un público inmigrante concreto. El perfil demográfico de las mujeres que respondieron la encuesta post-visita al Museo Marítimo, habla de una mujer joven, que lleva poco tiempo residiendo en Europa, que tiene algún tipo de estudio y que no habla ninguno de los dos idiomas oficiales de Cataluña: ni castellano ni catalán. Este fue, precisamente, uno de los principales problemas en la experiencia, durante la toma de datos y en el análisis de los resultados. Por ello, a pesar de haber sido una experiencia gratificante, los resultados no aportan ninguna conclusión clara respecto a su relación con el museo.

En el caso de Cosmocaixa, los visitantes manifestaron que conocer el museo les ayudó a conocer a la gente de Barcelona, a sus vecinos, a entender más a sus amigos de aquí, y al país en general. Hay que tener en cuenta que el perfil del visitante de Cosmocaixa nos muestra a un visitante especialmente latinoamericano y con estudios universitarios. Todo lo contrario al perfil de las mujeres que visitaron el Museo Marítimo. A pesar de ello, una minoría de las mujeres que visitaron el Museo Marítimo aseguró que les ayudó a entender a sus amigos catalanes

Hay que recordar que la encuesta del Museo Marítimo tuvo que simplificarse para que las encuestadas pudieran comprender las preguntas. Por ello, éstas no corresponden en su totalidad a las realizadas en Cosmocaixa. Al realizarse una toma de datos diferente, no se pretendió realizar una comparación ni cruzar los datos. No obstante, hay que resaltar que la mayoría de encuestados en las dos instituciones, encontraron elementos comunes a sus países de origen. En Cosmocaixa, especialmente nombrado fue el "Bosque inundado" y en el Museo Marítimo, los barcos pesqueros.

Como se apuntaba anteriormente, los resultados nos dan indicios de cómo se puede investigar el público inmigrante en nuestro país en cuanto a los museos científicos o con contenido científico se refiere, y cuan diversos son sus perfiles, lo que plantea un reto mayor para conocerlos y, en consecuencia, plantear acciones que atraigan a este grupo poblacional. Entre los muchos aspectos susceptibles de ser investigados, en cuanto a este colectivo se refiere, podemos destacar si los elementos comunes o los diferentes siguiendo a Stuart Hall, pueden ser puntos de partida para construir conocimientos y habilidades que formen parte de la identidad y de las identidades que construimos día a día.

- Fechas del proyecto de investigación: La investigación fue financiada por las tres instituciones participantes.

- inicio de la investigación: 11/2013

- término de la investigación: 04/2014 


\section{Notas}

1 El estudio preliminar contó con el apoyo de los dos museos, el grupo Museia i el Elearn Centre de la Universitat Oberta de Catalunya (UOC).

2 El "Bosque inundado" es la única reproducción que existe en Europa de bosque inundado de la selva amazónica de Brasil. Se trata de un espacio de $1.000 \mathrm{~m}^{2}$, uno de los espacios más visitados del museo.

\section{Referencias bibliográficas}

Barker, Ch. (2001): Cultural Studies. Theory and Practice. London (etc.): Sage.

Beck, U. (1992) : Risk Society. Towards a New Modernity. London: Sage.

Bourdieu, P. (1988) : La Distinción. Madrid: Taurus.

Castellanos Pineda, P. (2013): Museos como medios de comunicación: Aportes al cambio social desde los museos de ciencias. Actas del V Congreso Internacional Latina de Comunicación Social V CILCS - Universidad de La Laguna.

Castellanos Pineda, P. (2011): Aportes del patrimonio científico en la construcción de la identidad. Reflexión teórica. XII Reunión RED-POP. Brasil.

Castellanos Pineda, P. (2010): Comunicación pública de la ciencia y consumo cultural. La información científica como elemento diferenciador. Anuario Electrónico de Estudios en Comunicación Social "Disertaciones", 3 (2), Artículo 4.

Disponible en la siguiente dirección electrónica:

http://erevistas.saber.ula.ve/index.php/Disertaciones/

Dawson, E. (2014a) : Reframing social exclusion from science communication: Moving away from "barriers" towards a more complex perspective. Journal of Science Communication, 13(1), 1-5.

Dawson, E. (2014b) : 'Not designed for us': How science museums and science centres socially exclude low-income, minority ethnic groups. Science \& Education, 981-1008

Elizalde, A., Max-Neef, M. y otros (2002): Sociedad civil, cultura democrática e inclusión social. Xàtiva: L’Ullal Edicions.

Escobar, A. (2001): "Culture sits in places: reflections on globalism and subaltern strategies of localization”, in Political Geography (20) Pgs.139-174.

Fleming, Martha (2013): “Open minds- open doors". In Braendholt Lundgaard, I. \& Thorek Jensen, J. (2013): Museums social learning spaces and knowledge producing processes. Kopenhagen: Styrelsen Danish Agency for Culture, pp. 62-80 
Galla, A. (2013): "Benchmarking diversity in Museums". In Braendholt Lundgaard, I. \& Thorek Jensen, J. (2013): Museums social learning spaces and knowledge producing processes. Kopenhagen: Styrelsen Danish Agency for Culture, pp. 148-171.

Galbraith, J.K. (1973): La sociedad opulenta. Barcelona: Ariel.

García Canclini, N. (1997): "El malestar en los estudios culturales", Fractal n 6, julio-septiembre, 1997, año 2, volumen II, pp. 45-60.

McClafferty, T.; Rennie, L.; Groves, I. (1996): "Easy to use instruments for formative

evaluation", Evaluation and visitor research in museums special interest group, meeting. Museums Australia Conference. Sydney, Nova Gal-les.

Max-Neef, M. (2006): Desarrollo a escala humana. Barcelona: Icaria. Colección Antrazyt. $3^{\text {a }}$ edición.

Ministerio de Empleo y Seguridad Social. Observatorio Permanente de la Inmigración (2013): Extranjeros residentes en España. Extranjeros con Certificado de Registro o Tarjeta de Residencia en Vigor.

Documento electrónico:

http://extranjeros.empleo.gob.es/es/Estadisticas/operaciones/con-

certificado/201306/Residentes_Principales_Resultados_30062013.pdf

Moreno, J; Bruquetas, M. (2011): Immigració i Estat del benestar a Espanya. Barcelona: Obra Social "laCaixa".

Prazniak, D. \& Dirlik, A. (eds.) (2001): Places and politics in an age of globalization. Maryland: Rowman \& Littlefield.

Partners for Livable Communities (2011): Cultures Connect All. Rethinking Audiences in Times of Demographic Change. Washington: MetLife Foundation. Obra Social "la Caixa" (2012): Informe anual. Barcelona: laCaixa.

Accesible en:

http://obrasocial.lacaixa.es/deployedfiles/obrasocial/Estaticos/pdf/Informacion_corporativa/informe_ anual_2012_es.pdf

Rounds, J. (2006): “Doing identity work in museums”, in Curator, 49 (2). Pgs. 133-150.

Sandell, R. (2003). "Social inclusion, the museum and the dynamics of sectoral change", en Museum and Society, 1(1), pp. 45-62. Leicester: University of Leicester.

Sandell, R. (ed.) (2002): Museums, Society, Inequality. London, NY: Rutledge.

Silver, H. (1994). "Social exclusión and social solidarity: Three paradigms", en

International Labour Review. Vol. 133. Pg. 531-578. 
Sprünker, J. (2011): Educació patrimonial mitjançant recursos educatius en línia amb contingut de patrimoni cultural i xarxes d'aprenentatge. Tesi doctoral, Barcelona, juliol 2011.

\section{Artículos relacionados:}

Castellanos Pineda, P. (2009). "La comunicación pública de la ciencia y los procesos de inclusión social. El caso de los museos de ciencias y la población vulnerable”, en VII Bienal Iberoamericana de la Comunicación. En actas del congreso. Chihuahua, México: Universidad Autónoma de Chihuahua.

Castellanos Pineda, P. (2008). Los museos de ciencias y el consumo cultural. Una mirada desde la comunicación. Barcelona: Editorial UOC

Sprünker, J.; Munilla, G.; Castellanos, P. (2014). "Educación patrimonial en línea: patrimonio accesible, inclusión intelectual y social del alumnado de secundaria obligatoria”, en Domínguez Arranz, A.; Juan García Sandoval, J. y Lavado Paradinas, P. (eds.)

II Congreso Internacional de Educación y Accesibilidad en Museos y Patrimonio: En y con todos los sentidos, hacia la integración social en igualdad. Actas. Huesca: Universidad de Zaragoza. T. 1, pg. 177-188

Tilly, A.; Gewirtz, Sh., and Cribb, A. (2007). "New labour's socially responsible museum. Roles, functios and greater expectatios", in Policy Styudies. Vol. 28, № 3. Pg. 269-289. Routledge.

\section{Cómo citar este artículo / Referencia normalizada}

P Castellanos Pineda, J Sprünker Cardó, G Munilla Cabrillana (2016): “Inmigrantes y museos de ciencias en tiempo de crisis. Estudio preliminar en dos museos de Barcelona". Revista Latina de Comunicación Social, 71, pp. 570 a 591

http://www.revistalatinacs.org/071/paper/1110/30es.html

DOI: $10.4185 /$ RLCS-2016-1110

- En el interior de un texto:

... P Castellanos Pineda, J Sprünker Cardó, G Munilla Cabrillana (2016: 570 a 591)...

$\mathrm{O}$

...P Castellanos Pineda et al., 2016 (570 a 591)...

Artículo recibido el 28 de abril de 2016. Aceptado el 18 de junio.

Publicado el 29 de junio de 2016. 
Revista Latina de Comunicación Social \# 071 - Páginas 570 a 591

\begin{tabular}{l|l|l|l|l} 
Investigación & DOI: 10.4185/RLCS-2016-1110 & ISSN 1138-5820 & Año 2016
\end{tabular} 